\title{
ORGANISATION SPATIALE D'UN ÉCOSYSTĖME EXPLOITÉ : LES CHOIX SPATIAUX DANS LA MODÉLISATION INTÉGRÉE DU DELTA INTÉRIEUR DU NIGER AU MALI
}

\author{
Yveline Poncet, Christian Mullon, Marcel Kuper
}

Belin | « L'Espace géographique »

2002/2 tome 31 | pages 118 à 130

ISSN 0046-2497

ISBN 2701131278

Article disponible en ligne à l'adresse :

https://www.cairn.info/revue-espace-geographique-2002-2-page-118.htm

Distribution électronique Cairn.info pour Belin.

(C) Belin. Tous droits réservés pour tous pays.

La reproduction ou représentation de cet article, notamment par photocopie, n'est autorisée que dans les limites des conditions générales d'utilisation du site ou, le cas échéant, des conditions générales de la licence souscrite par votre établissement. Toute autre reproduction ou représentation, en tout ou partie, sous quelque forme et de quelque manière que ce soit, est interdite sauf accord préalable et écrit de l'éditeur, en dehors des cas prévus par la législation en vigueur en France. Il est précisé que son stockage dans une base de données est également interdit. 


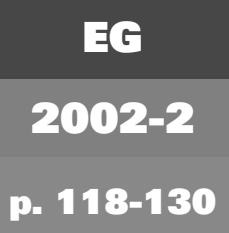

\section{Yveline Poncet, Christian Mullon, Marcel Kuper \\ IRD, Centre d'Orléans, 5 rue du Carbone, 45072 Orléans cedex 2}

RÉSUMÉ .- Dans la région très plane et inondée saisonnièrement qu'est le delta malien, la dynamique de l'eau conditionne directement et indirectement les processus sociaux et techniques de la production, et pèse sur la plupart des perspectives de développement, à toutes les échelles : la circulation de l'eau est le moteur de l'écosystème. L'organisation spatiale qu'elle définit est l'élément de base d'un modèle intégré des processus physiques, biologiques et sociaux en jeu dans le delta. Nous présentons ce modèle en mettant l'accent sur la façon dont y est traitée la dimension spatiale. La place qui lui a été accordée a permis de développer une interface concrète et visible sous la forme de cartes statiques et cinétiques, entre les concepts, les données et les mécanismes qui constituent le modèle.

DELTA, EAU, ÉCOSYSTEME, MALI, MODÉLISATION SPATIALE
ABSTRACT . - Spatial organisation of an exploited ecosystem: spatial choices in the integrated model of Mali's interior delta of the Niger River. - In the Mali Delta, a flat region that is seasonally flooded, the dynamic of water directly and indirectly conditions the social and technical processes of production and influences most development prospects, on all scales: the circulation of water is the motor of the ecosystem. The spatial organisation defined by water is the basic element in an integrated model of the physical, biological and social processes at work in the delta. In our presentation of this model, we emphasise the way it deals with the spatial dimension. This emphasis on the spatial dimension has made it possible to develop a concrete, visible interface, in the form of statistical and kinetic maps, between the concepts, the data and the mechanisms that constitute the model.

DELTA, ECOSYSTEM, MALI, SPATIAL MODELLING, WATER

\section{Introduction}

Le delta intérieur du Niger mesure un peu plus de $30000 \mathrm{~km}^{2}$ et abrite un million d'habitants, surtout pêcheurs, agriculteurs, éleveurs. Leur spécialisation professionnelle et sociale dans l'exploitation de plusieurs ressources naturelles renouvelables a conduit à des formes originales d'organisation territoriale et à des partages raisonnés et efficaces de l'espace et du calendrier de production, non sans conflits cependant (Gallais, 1967; Fay, 1989 et 1997; Barrière et Barrière, 1995; Poncet et Orange, 1999). Le gradient hydroclimatique entre amont et aval, le compartimentage géomorphologique induit par plusieurs millénaires d'accumulation et d'érosion fluviales, les irrégularités interannuelles du cycle hydrologique ont engendré une grande diversité géographique et temporelle des milieux, dont tirent parti les systèmes de production. Un même site peut être successivement rizière, pâture et lieu de pose d'engins de capture du poisson, 
avec des ayants droit et des règles d'accès différents. Cette variabilité s'observe au niveau de la région naturelle inondable dans son ensemble (niveau régional) : écoulement des eaux, transhumance des éleveurs, déplacements des pêcheurs avec la décrue; elle s'observe aussi au niveau local de la gestion des terroirs par les communautés villageoises: connaissances paysannes sur les ressources, droits et contrôles sur leur prélèvement, maîtrises territoriales, gestion des voisinages.

Or, si les gestions traditionnelles des ressources naturelles renouvelables du delta paraissent avoir été jadis à la fois conflictuelles et fonctionnelles, les transformations (politiques, sociales, économiques, administratives) subies à partir des années 1920 ont été accompagnées de pertes d'efficacité et de crises (Fay, 1989; Quensière et al., 1994) : simplification des savoirs, disparition de la légitimité des maitrises et des contrôles locaux, difficultés croissantes à résoudre des conflits qui ne sont pas tous fonciers, interventions maladroites de l'État, baisse ou stagnation de certaines productions économiques. Ces dégradations ont été mises en évidence et aggravées par les pertes de productivité naturelle pendant les années de sécheresse, entre 1972 et 1994.

Nous avons développé un modèle intégré du delta intérieur du Niger pour montrer où, quand et sur quels processus se manifeste la complexité des interactions naturelles et sociales en jeu, et pour simuler l'impact d'événements et de décisions sur les systèmes de production ${ }^{1}$. L'objectif est de répondre à des questions communes aux acteurs du développement et aux producteurs eux-mêmes : comment ce système organisé va-t-il évoluer sous l'action de phénomènes raisonnablement prévisibles accroissement démographique, changement climatique, évolution technique, aménagements hydrauliques et agricoles?

\section{Les niveaux d"organisation}

Le renouvellement des fertilités naturelles du delta et les actions humaines directement liées à la production de riz, de bétail et de poisson sont principalement liées aux superficies inondées (Gallais, 1967; Laë, 1992), secondairement à la durée de l'immersion des terres et aux vitesses de montée puis de descente de l'eau. Les tracés hydrographiques et leurs organisations, d'une part, les débits, d'autre part, structurent donc le système régional (l'écosystème exploité) aussi bien que les systèmes locaux (de l'ordre de plusieurs dizaines à plusieurs centaines de kilomètres carrés). En conséquence, le modèle intégré du delta intérieur du Niger est fondé sur l'hydrographie, l'hydrologie et les formes du relief comme facteurs d'organisation. L' "eau", thème directeur, sert de base à l'architecture du modèle, à laquelle se réfèrent les données et les mécanismes des autres thèmes. Sa construction géométrique dirige tout le modèle.

\section{Le niveau régional}

L'espace de la région naturelle correspond sur le terrain au périmètre extérieur du niveau des crues fluviales atteint au cours de la décennie 1950 (qui sont parmi les plus abondantes et les plus constantes du siècle), tel que figuré sur la carte topographique régulière de l'AOF au 1:200000. Dans le modèle, il est transcrit comme un réseau orienté et ouvert, constitué de plusieurs entités hydrographiques de forme simple, géoréférencées.

L'espace de l'eau est construit comme un compromis entre trois contraintes: le respect des multiples dynamiques hydrauliques (déversements, interruptions, hystérésis...) ; la prise en compte des objets hydrographiques au plus près de leur organisation
1. Ce modèle est accessible sur le site internet :

http://www.orleans.ird.fr 


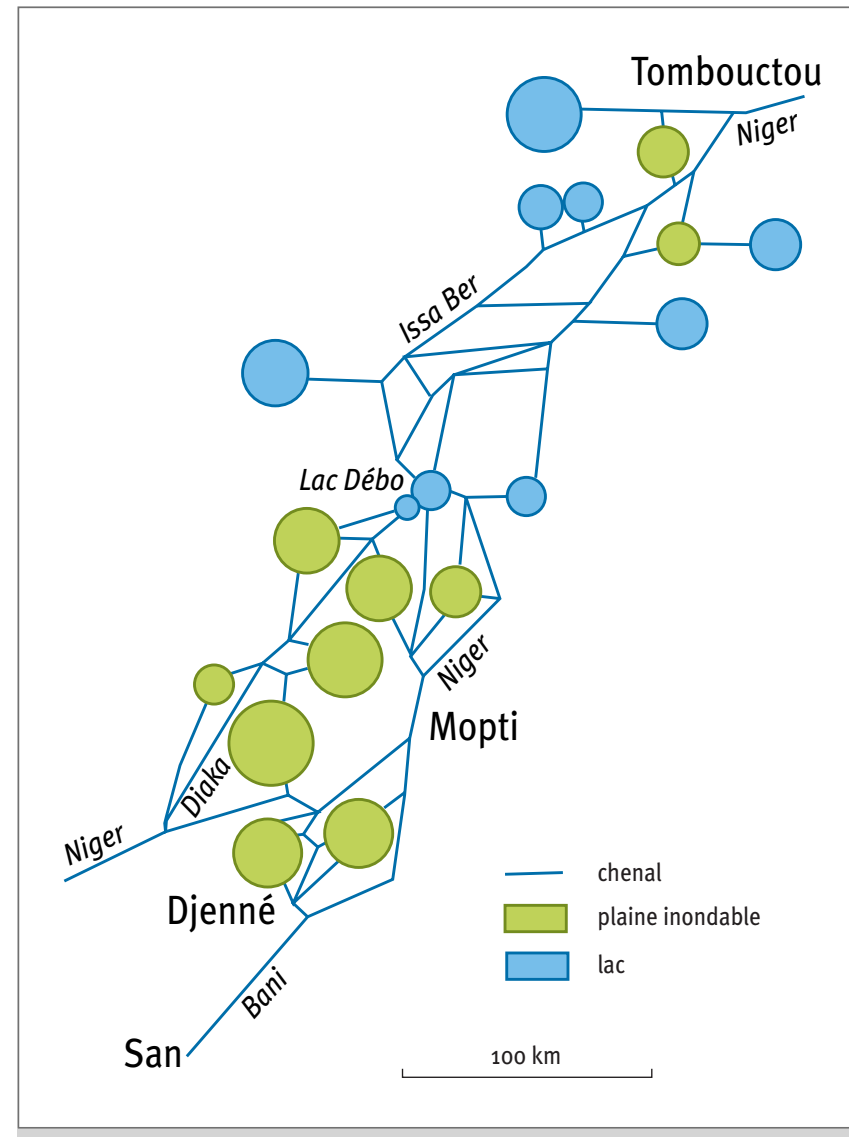

Fig. 1/ Le schéma spatial du delta intérieur géographique réelle; la schématisation des tracés fluviaux et des typologies hydrographiques, afin de présenter une image du delta clairement lisible dont chaque élément soit identifiable.

Le résultat de ce compromis est une construction géométrique qui transcrit un modèle fluxstockage (fig. 1). Les flux sont supportés par des segments de droite délimités par des connexions; ils correspondent aux biefs du fleuve Niger et du Bani et à leurs chenaux adjacents, affluents et défluents. Tous sont aisément repérables et identifiables (localisation, tracés, noms) sur les cartes au $1: 200000$. Les réservoirs sont schématisés par des cercles, qui correspondent aux lacs (stockage permanent) et aux plaines inondables qui s'assèchent complètement en étiage (stockage temporaire). L'organisation relative des 109 entités spatiales retenues respecte les distances entre les connexions et les dissymétries de l'hydrosystème, qui gouvernent en partie les gradients temporels: la distance entre l'entrée et la sortie du delta et la présence des lacs centraux à mi-parcours retardent les flux; l'inégale répartition des plaines inondables entre l'amont et l'aval des lacs centraux, entre la rive gauche et la rive droite, contribue aussi aux différentiels temporels (date du maximum de la crue, durée de la décrue). On a donc placé les connexions et les centres des cercles à une place géographique précise, au plus près de l'exactitude en latitude et longitude. L'un des avantages de cette option est que le schéma géométrique est superposable aux cartes et immédiatement reconnu par les utilisateurs. Le repérage entre le réel et la représentation spatiale schématique est rendu plus aisé encore par la toponymie réelle des entités dans les fichiers et l'interface d'utilisation.

Le niveau régional est donc constitué par l'ensemble des entités hydrographiques choisies comme nécessaires et suffisantes pour rendre compte du fonctionnement dynamique de l'ensemble de la région naturelle. Le périmètre extérieur de la région inondable n'y figure pas, le phénomène géographique régional de l'inondation est transcrit par la somme des superficies inondées des entités spatiales. Cette somme reste un espace discontinu et non pas une tessellation: on a résolument privilégié ici le réseau et le processus flux-stockage. Nous avons préféré un modèle spatial discret, en réseau, à un modèle spatial continu, en zones, afin de respecter l'objectif de l'opération: montrer l'hydrosystème exploité dans son intégralité, afin de proposer un cadre unique pour le gérer comme un tout.

Nous suivons une démarche de modélisation exploratoire, partant d'hypothèses aussi simples et explicites que possible sur le fonctionnement du système, qui formalise les processus choisis et permet la comparaison des dynamiques simulées avec les dynamiques observées, représentées à partir des approches descriptives antérieures. 
C'est à ce moment que nous nous référons à la mosaïque continue de thématiques que fournissent les cartographies existantes ${ }^{2}$ et les approches plus dynamiques obtenues avec un système d'information géographique (Marie, 2000 et 2001).

\section{Le niveau local}

Chaque entité spatiale est construite pour intégrer les attributs hydrologiques, hydrobiologiques, agronomiques, socio-économiques de son fonctionnement local. Les chenaux sont délimités par leurs connexions. Celles-ci ont un sens hydrologique (perte par déversement, apport par affluence). Elles ont aussi un sens social: les points de confluence et de défluence ont joué un rôle dans l'organisation des maîtrises sur l'eau et donc sur les organisations politiques et sociales d'aujourd'hui; ils délimitent des biefs auxquels correspondent des statuts fonciers, des règles d'usage, des alliances, des enjeux de production.

La délimitation des plaines inondables, en revanche, est moins directement lisible puisque les cercles qui les représentent intègrent plusieurs unités topographiques perçues sur le terrain comme distinctes. La notion de plaine inondable recouvre un ensemble compliqué, parfois variable, souvent flou, de compartiments bas à des altitudes différentes, délimités par les bourrelets de berge de leurs affluents et émissaires. Les plaines ne présentent pas à l'observateur de limites aisément repérables. Elles sont cependant bien identifiées par leurs paysans, qui connaissent leurs sols, leur végétation naturelle, les ressources exploitables et le rythme de leurs submersions et assèchements. Chaque entité spatiale "plaine» restituée dans le modèle intègre plusieurs plaines paysannes, connexes et en intercommunication hydrologique. Leur connexité même leur donne des traits biophysiques communs, transcrits avec plus ou moins d'intensité par certains traits sociaux communs ${ }^{3}$.

Les entités spatiales sont le support géométrique des zones de production qui sont construites en rectangles à partir des segments et conservent les cercles. Ces zones contiennent les lieux de la production: sites de pêche, rizières, pâturages, agglomérations d'habitat des producteurs. Elles ne sont pas conçues pour que leur figuration remplisse un espace continu ${ }^{4}$, ce qui est conforme à la réalité du delta, dans lequel, d'une année à l'autre, d'une saison à l'autre, tous les espaces ne sont pas productifs.

Dans chaque entité spatiale, les trois systèmes de production sont considérés comme mettant en jeu deux processus situés à l'amont et à la source de la production économique elle-même: la production naturelle de ressources, fonction des superficies calculées d'après les paramètres hydrologiques d'inondation spécifiques à chaque entité spatiale; la disponibilité des exploitants pour le prélèvement, fonction du nombre de producteurs présents, les uns, sédentaires, recensés dans une entité en permanence et géographiquement attachés à elle, les autres, mobiles, rattachés à une entité et en fréquentant d'autres de façon temporaire.

Les entités spatiales géométriques, qui sont aussi hydrographiques et hydrologiques (Poncet et al., 2001), sont donc les unités d'espace élémentaire du modèle. Chacune d'entre elles a un sens local et un rôle dans l'ensemble régional et permet de mettre en œuvre un même principe de connectivité à tous les objets thématiques du modèle: circulation de l'eau, succession des droits de pêche d'amont en aval, déplacements de main-d'œuvre, transhumance des troupeaux. Elles accueillent les producteurs, organisés en groupes: communautés villageoises entières ou segmentées, familles entières ou segmentées, classes d'âge. Ces groupes se divisent et se réunissent selon les
2. Programmes PIRT (1983) et PIRL (1990) entre autres.

3. C'est ainsi que les plaines (au sens paysan) connexes et les rives de leurs chenaux adjacents constituent souvent une même " propriété " pastorale ou un " pays halieutique " (Poncet et Quensière, 1996; Poncet, 2000) exploité par plusieurs villages liés entre eux par des échanges matrimoniaux.

4. Mais si I'on veut rapporter les zones dans leur ensemble à la superficie du delta, on constate que leur superficie moyenne est de $275 \mathrm{~km}^{2}$ environ, ce qui correspond à la dimension de plusieurs des "pays" (ou communautés plurivillageoises) identifiés sur le terrain. 
occasions offertes par l'inondation, en fonction des droits qu'ils détiennent ou qu'ils négocient dans telle entité sur le prélèvement des ressources qui s'y trouvent réellement. Ces droits s'exercent rarement sur l'espace uniquement; ils s'interprètent plus souvent à la fois sur la ressource, sur l'espace et dans le temps, en termes de dates, durées et préséances. Les entités-acteurs en jeu dans le processus de production correspondent ainsi à des groupes de pêcheurs et de riziculteurs et à des troupeaux de bovins. Ce ne sont pas les pêcheurs, les riziculteurs ni les éleveurs qui sont représentés avec leurs stratégies économiques (investissement, production, commercialisation), ce sont des groupes avec des comportements (migration, actes de la production) tels qu'ils sont connus d'après les travaux de terrain et les enquêtes (Daget, Gallais, Quensière, Kuper).

Ces deux échelles géographiques (régionale et locale) suffisent à inscrire et à restituer les fonctionnements naturels et sociaux. Le groupe de producteurs, de dimension variable et toujours rapporté à une entité spatiale, permet de conserver l'intégrité géographique du modèle: aucun producteur ne peut se trouver dans deux entités différentes en même temps.

\section{Les mécanismes physiques et biologiques du modèle}

Tous les mécanismes inscrits dans le modèle font intervenir l'espace de chaque entité, et donc, par les connexions qu'elles entretiennent, l'espace de l'ensemble du delta.

\section{Le renouvellement des ressources}

La production naturelle de ressources renouvelables (fertilité des sols cultivables, herbages, poissons) dans chaque entité spatiale est calculée en fonction de sa superficie inondée, d'après des modèles issus des travaux antérieurs, affinés au cours de la construction de la modélisation intégrée. Le processus physique qui lie les débits fluviaux aux superficies inondées dans toutes les entités est restitué par un modèle de vases communicants qui fait intervenir la profondeur de chaque entité et les altitudes relatives de leurs connexions (Kuper et al., 1999 et 2000) : la hauteur d'eau (fournie par des mesures sur le terrain et interpolée à chaque entité spatiale) induit la superficie inondée des zones correspondantes, schématisées comme des cônes (plaines et lacs) et comme des parallélogrammes obliques (chenaux). Les mécanismes qui permettent de calculer la productivité naturelle de chaque zone s'inscrivent alors dans chaque surface inondée.

Deux équations rendent compte de la productivité naturelle de poissons: la reproduction est calculée dans les plaines inondables, suivant une équation classique de dynamique des populations dans laquelle la capacité d'accueil dépend de la superficie inondée; la diffusion du poisson est calculée ensuite suivant une équation de mélange fonction de la connectivité entre les zones.

Les superficies réellement productrices de riz sont directement déduites des superficies inondées dans les plaines. Sont prises en compte uniquement les cultures de riz traditionnel (riz à submersion libre, riz de décrue), qui dépendent de la qualité de l'inondation (Kuper et Maiga, 2000; Maiga et al., 2000). Les agriculteurs se déplacent d'une zone agricole à une autre en fonction de la surface inondée et des connexions sociales qu'ils entretiennent avec les zones de production. Pour chaque zone agricole et chaque quinzaine de jours, les besoins en main-d'œuvre sont calculés 
selon la saison: labour et semis; désherbage, sarclage; récolte et battage. Le calendrier des activités et la charge de travail sont principalement basés sur les travaux de Gallais (1967 et 1984) et les observations des auteurs (à paraître). La surface de riz ensemencée dans une zone pour une année donnée dépend de la surface agricole disponible dans cette zone, de la quantité de main-d'œuvre et des ressources en semences. Elle ne dépend pas de la crue de l'année, car le semis se fait avant la montée des eaux. En revanche, les travaux d'entretien et de récolte dépendent directement des conditions hydroclimatiques: les riziculteurs récoltent assez souvent sur des surfaces moitié moindres que celles qu'ils ont ensemencées. La production de riz est obtenue en multipliant les superficies récoltées par le rendement.

La productivité potentielle des pâturages est calculée en fonction de la hauteur d'inondation, laquelle définit le type de végétation (d'après CIPEA-ODEM, 1983; Kuper et al., 1999 et 2000) : la prise en compte de la profondeur d'inondation permet de tenir compte des considérables variations de qualité et de quantité de pâturage, qui vont du simple au quintuple.

\section{Les systèmes de production}

La prise en compte de la spatialité des comportements est essentielle. Les processus qui conduisent de la ressource naturellement produite au produit économiquement transférable (du poisson vivant au poisson pêché, par exemple), sont restitués par un modèle d'attraction (pulling model) pour la pêche et la riziculture, et par un modèle de chasse (pushing model) pour l'élevage.

Les zones offrent des ressources différentes dans l'espace et dans le temps, et les groupes de pêcheurs et de riziculteurs tirent parti de ces différences pour prélever des ressources rendues plus ou moins abondantes par l'inondation, au lieu et au moment opportuns. Ces groupes d'acteurs se déplacent ainsi d'une zone à une autre en fonction de la productivité des zones (c'est-à-dire de leur superficie inondée) et en fonction des liens sociaux et politiques qui leur donnent, dans telle ou telle zone, des droits d'exploitation. Un même groupe d'acteurs peut donc prélever (produire) successivement dans plusieurs zones.

La question des droits d'exploitation dans le modèle pastoral, les spécificités de la transhumance sahélienne et de l'élevage bovin dans le delta ${ }^{5}$ ont conduit à chercher des solutions originales pour en rendre compte dans le modèle tout en conservant la même architecture spatiale et les mêmes principes de mécanisme que pour la pêche et la riziculture. Ici, les acteurs-préleveurs de la ressource en herbe sont les troupeaux, qui se déplacent d'une zone à l'autre sous deux contraintes: un calendrier qui est à la fois naturel (décrue, croissance de l'herbe) et sociopolitique (réglementations séculaires mises en place par un pouvoir solidement légitimé) ; la diminution progressive de la capacité de charge des zones: lorsqu'elle est épuisée par une pression trop élevée, une zone chasse les troupeaux qui s'y trouvent vers les zones voisines autorisées par le calendrier.

Plusieurs groupes peuvent se succéder (voire coexister) sur la même zone selon leurs droits, les uns historiquement détenus (groupes propriétaires), les autres négociés à court ou moyen terme (groupes étrangers). La structuration spatiale à deux niveaux et les mécanismes du modèle schématisent et résument des phénomènes naturels, des attitudes sociales, des processus de production qui se déroulent et s'observent, dans la réalité grandeur nature, à des échelles multiples et mouvantes.
5. Entre autres particularités, si on le compare aux autres systèmes pastoraux sahéliens: disponibilité en eau d'abreuvement presque illimitée, calendriers de transhumance très précis et (en principe) contraignants, domination historique, politique et foncière par les éleveurs jusqu'à et $y$ compris la période actuelle. 


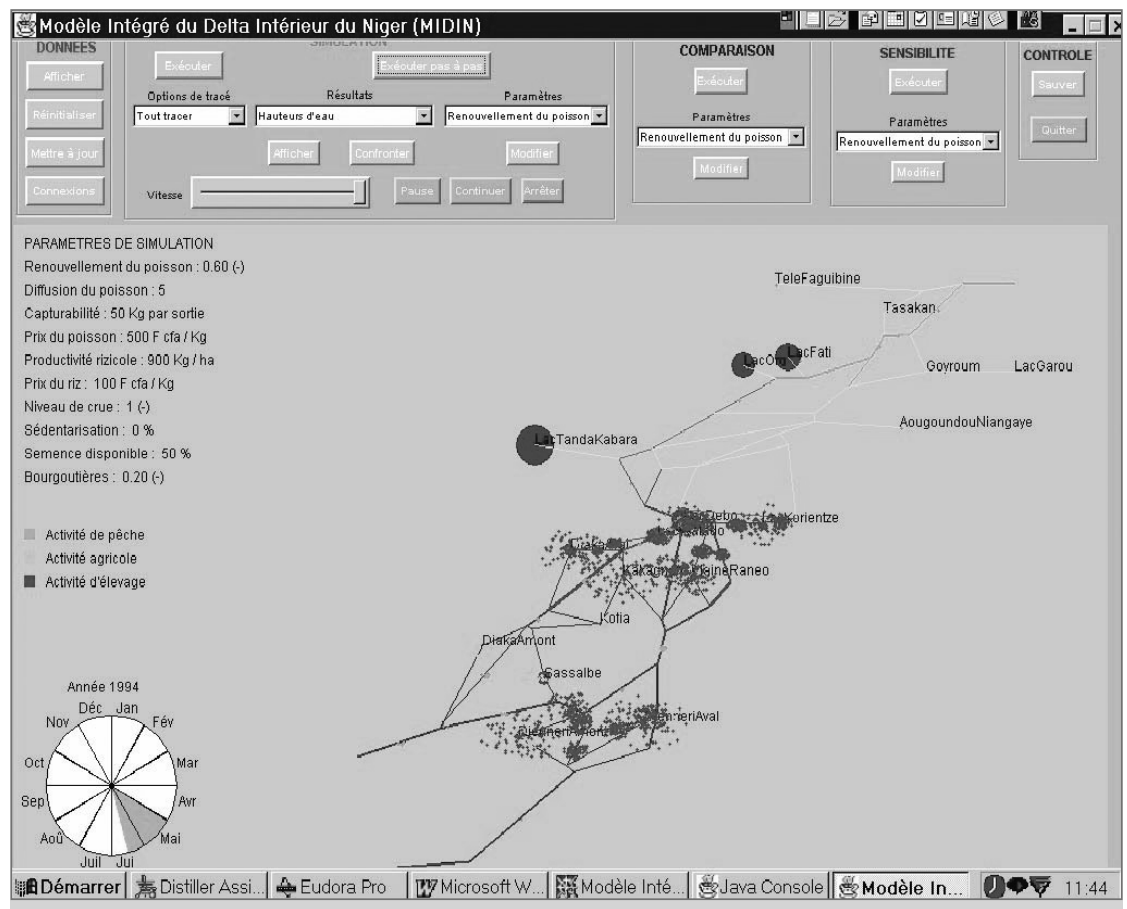

Fig. 2 / L'un des 72 états de la carte cinétique de simulation, milieu du mois de juillet en année déficitaire

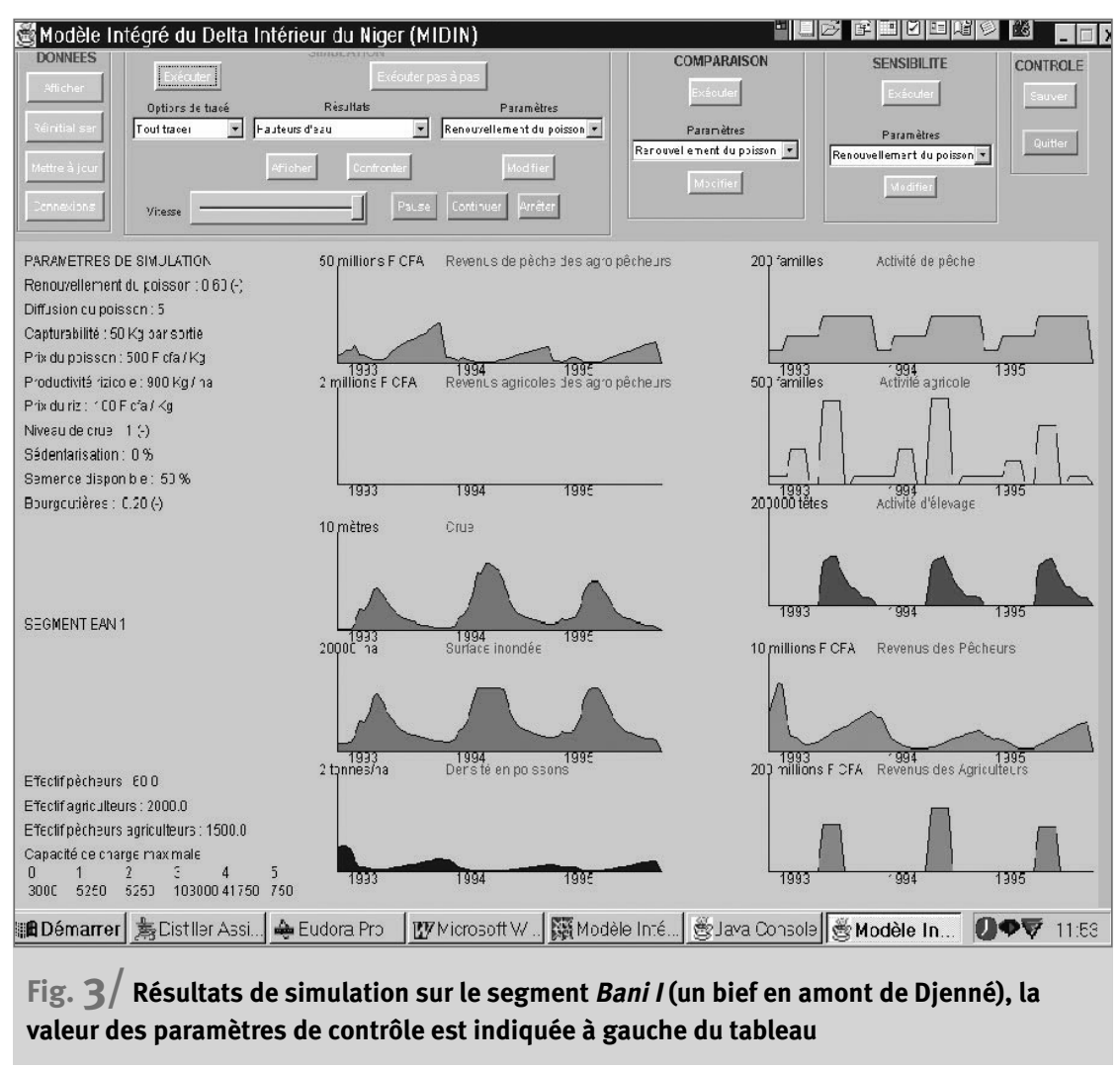




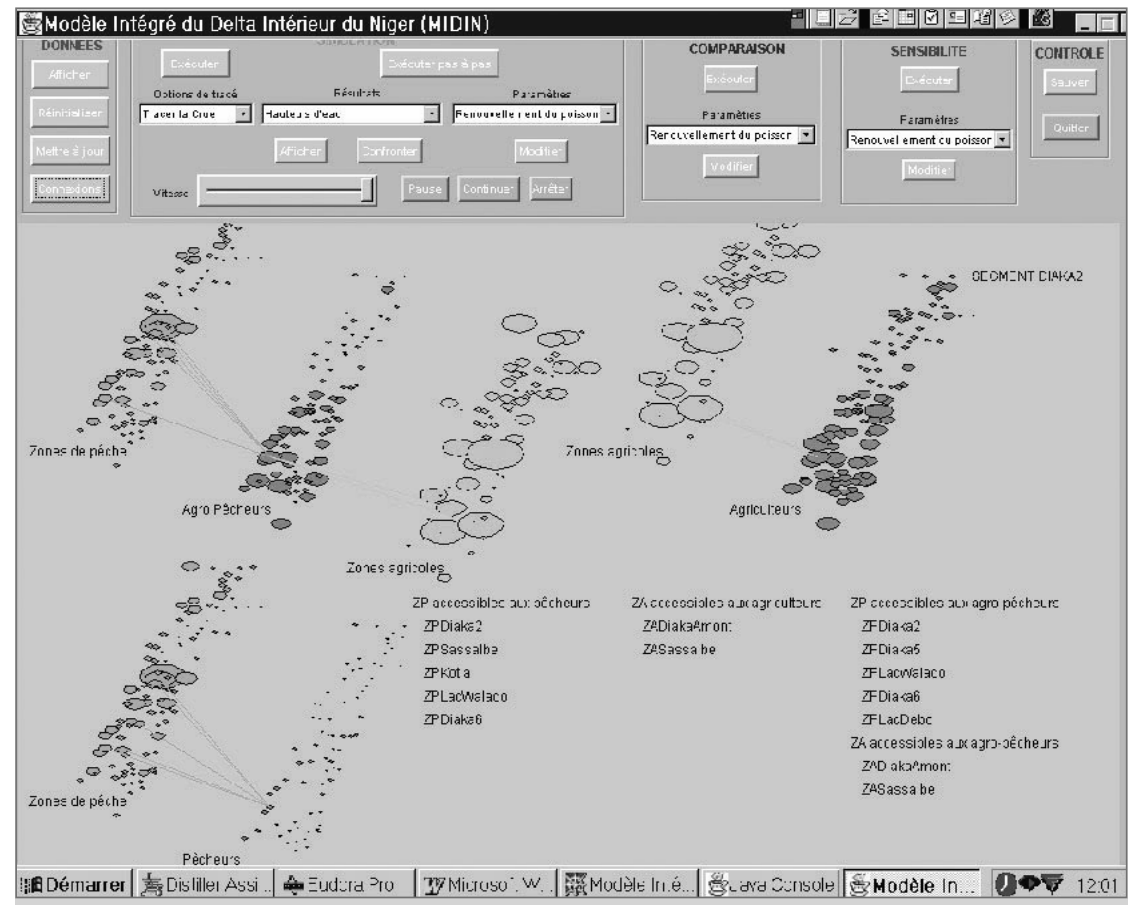

Fig. 4/ Les connexions spatiales entre zones de résidence des producteurs (agriculteurs et pêcheurs) et zones de production agricole (ZA) et de pêche (ZP) concernant un bief du Diaka

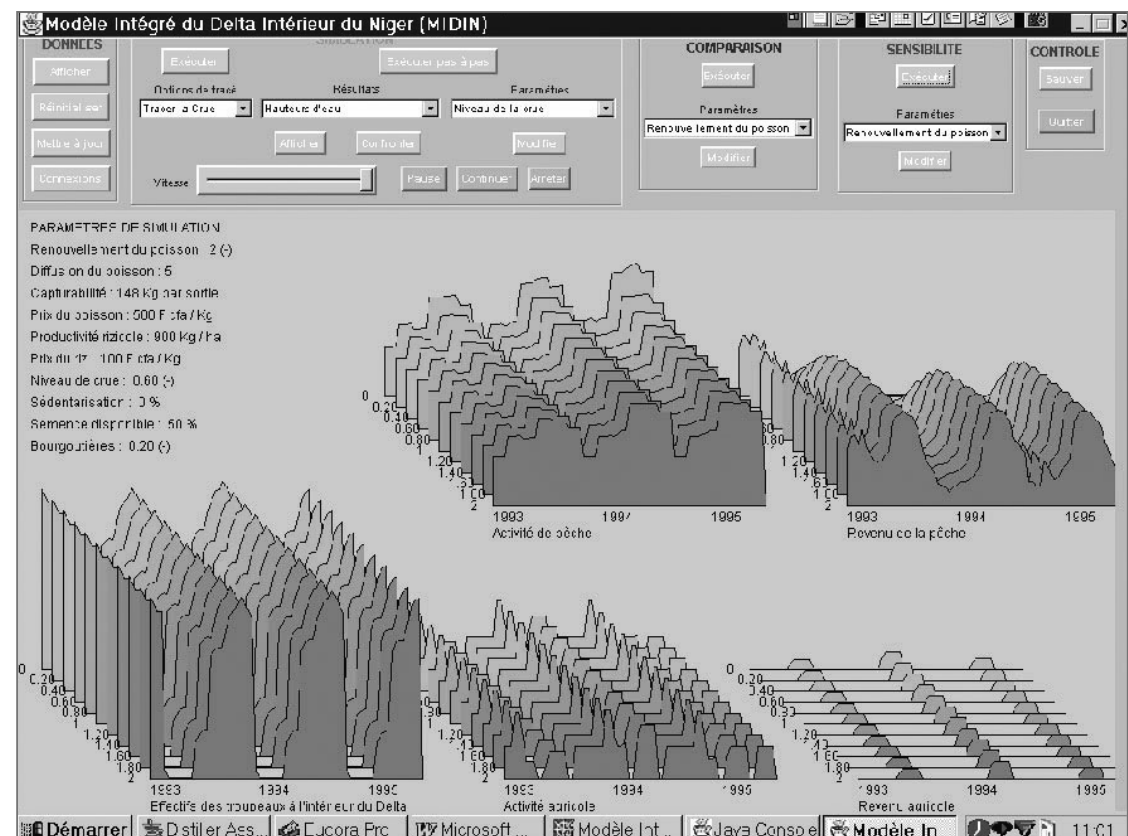

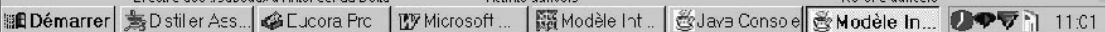

Fig. 5/ Analyse de sensibilité. En cas de capturabilité élevée du poisson (ici 148 kg par sortie) et de crues peu abondantes (ici indice 0,60 ), on teste la sensibilité du système à la variation de renouvellement naturel du poisson. 


\section{Le simulateur}

\section{Contrôle des simulations}

La fonction simulation de la modélisation intégrée du delta intérieur du Niger propose (dans la version 2001) 12 paramètres de contrôle dont 7 sont concevables et transcriptibles dans l'espace: l'importance de la crue par rapport aux hauteurs réellement observées et inscrites; la vitesse avec laquelle les poissons se dispersent dans tout l'espace disponible, qui est notamment fonction des micro-aménagements (creusements, diguettes) pratiqués par les pêcheurs et les agriculteurs; le rendement à l'hectare du riz paddy; la mobilité des pêcheurs et des riziculteurs; la superficie relative de bourgou 6 disponible, que l'action de l'homme augmente (repiquages, réintroductions), ou diminue (défrichements au profit des rizières) ; la capacité du réservoir d'un aménagement hydraulique en amont du delta; l'altitude de la cote de retenue d'un aménagement hydraulique en aval du delta. Les variations de valeur de ces paramètres permettent de simuler des transformations de l'espace du delta concernant l'ensemble régional, les relations entre zones, ou telles zones particulières.

\section{Présentation des résultats}

Les résultats des simulations sur des scénarios proposés par l'équipe scientifique et par les utilisateurs du modèle sont présentés principalement sous la forme d'une carte cinétique du delta, qui reproduit les états du système sur trois ans, avec un pas de 15 jours, soit une succession de 72 états (fig. 2). Les hauteurs d'eau des trois années ont été choisies pour parcourir trois situations couramment observées: une année déficitaire, une année excédentaire, une année moyenne ${ }^{7}$. Les données hydrologiques sont celles de la série réelle 1993-1994, 1994-1995, 1995-1996, qui obéit à la condition ci-dessus. Chaque entité spatiale peut être interrogée séparément, les résultats s'affichent alors sous forme de courbes, chacune figurant en abscisse les 3 années de la simulation (fig. 3). Les connexions spatiales entre zones productives peuvent être affichées (fig. 4). Le déroulement des simulations peut être suivi pour chaque thématique à travers des animations graphiques. Enfin, les analyses de sensibilité restituent la réponse de toutes les zones dans des diagrammes à trois dimensions (fig. 5). Des simulations précises sur des hypothèses proposées par les utilisateurs du modèle sont présentées et commentées dans plusieurs documents (cf. Kuper et al., 1999 et 2000).

\section{Validation}

La validation du modèle exploratoire d'un système complexe, qui intègre phénomènes biophysiques et phénomènes sociaux, ne peut pas être faite de façon classique en comparant les résultats du modèle avec ceux du terrain ou ceux d'une expérience.

6. Echinochloa stagnigna et Vossia cuspidata, le pâturage excellent du delta.

7. Calcul des moyennes effectué sur la série 1960-1997.

8. Directions des services techniques ministériels, enseignants, ONG de développement, chercheurs de I'Institut d'économie rurale du Mali et de I'IRD. Suivant Kuper (1997) et de Marsily (1997), nous avons considéré trois critères permettant, non pas de valider le modèle, mais plutôt de le justifier, et qui nous guident dans notre démarche. Ces trois critères sont:

- la plausibilité: les dynamiques obtenues doivent être vraisemblables; ce point a été testé

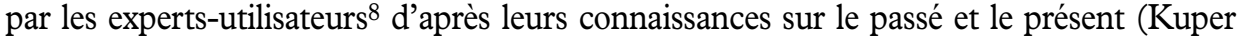
et al., 1999 et 2000) sur des secteurs géographiques précis qui leur sont bien connus;

- la reconstitution, à un niveau d'ensemble, d'événements historiques notables; c'est pour cette raison que nous avons basé le modèle sur l'hydrologie des années 1994 à 1996, qui sont très contrastées et dont la dynamique a été étudiée en détail; 
- la possibilité de conduire des analyses de sensibilité : le modèle privilégie un ensemble de paramètres de contrôle (intensité des crues, capturabilité du poisson, fertilité, disponibilité des semences, etc.) et offre la possibilité d'étudier les effets des variations d'un de ces paramètres sur la dynamique du système.

Il nous apparaît maintenant que la plausibilité spatiale du modèle est assurée par plusieurs facteurs: le géoréférencement des entités restitue une image réelle et reconnue, à la fois dans l'ensemble régional et entité par entité; les entités spatiales, dans leur nature et dans leur nom, sont des objets familiers à tous les acteurs du développement et de la décision dans le delta: leurs actes (qu'ils soient de production de riz ou de réglementation des transports) et leurs savoirs sont couramment référencés par eux-mêmes sur les mêmes lieux, aux mêmes niveaux; la reconnaissance des localisations et des formes à travers l'interface spatiale est le support des échanges entre les acteurs sur le fonctionnement du delta et des discussions sur les alternatives de gestion; plus particulièrement, la mobilité des ressources et des producteurs est clairement figurée, ce qui montre la complexité des relations entre les multiples pays du delta, ainsi que les liens traditionnels des producteurs avec ces différents pays.

\section{Discussion}

\section{Un modèle réticulaire}

Le choix de modélisation réticulaire flux-stockage comme base spatiale cohérente $d u$ modèle tout entier rend bien compte de la dynamique de l'écosystème : à tout moment de l'année, il y a flux et stockage et non pas flux uniquement ou stockage uniquement. Le delta en hautes eaux, par exemple, n'est pas un immense réservoir mais une combinaison de dynamiques différentes. Cette présentation permet de mettre en évidence la cause première des disparités géographiques à l'intérieur du delta. En effet, le but du modèle n'est pas d'identifier (délimiter, cartographier) des secteurs géographiques différents, mais de montrer pourquoi et comment les milieux se différencient en fonction de la crue. Cette approche a emporté l'adhésion des utilisateurs: la schématisation géométrique du delta, le traitement de l'espace dans l'écriture des mécanismes, l'affichage localisé et cartographié des résultats paraissent familiers en même temps qu'originaux, compréhensibles et efficaces.

Le modèle réticulaire flux-stockage permet également de contourner l'absence de modèle numérique de terrain: dans une région aussi plane et aussi étendue, dans laquelle les micro-reliefs de l'ordre du centimètre et du décimètre ont autant d'importance, cette lacune risque de ne pas être comblée avant très longtemps ${ }^{9}$.

La spatialisation paraît bien être une voie utile pour construire la présentation d'un système complexe dans lequel interagissent des phénomènes de nature différente. Les dimensions géographiques du système naturel et les multiples niveaux et critères d'organisation des systèmes sociaux ont rendu cette approche indispensable. L'espace est en effet l'un des supports à la fois matériel et conceptuel qui permet de superposer, de combiner et de montrer des processus différents concernant des objets différents inscrits dans le même réseau. Le fait que ce réseau soit connu et lisible (dans le delta et au Mali en général) permet que ses espaces deviennent, par le modèle, supports et objets des dialogues et des négociations entre tous les acteurs du développement.
9. Le système d'information géographique Delmasig (Marie, 2001) permet lui aussi et dans une certaine mesure de s'en affranchir, grâce à ses classes de hauteurs d'eau et ses capacités d'analyse spatiale. 
Il faut cependant préciser que la modélisation intégrée du delta du Niger est le résultat de travaux scientifiques localisés entrepris depuis longtemps et qui se sont poursuivis sur plusieurs dizaines d'années en approfondissant chaque système de production: les signatures de Gallais, Daget, du CIPEA et de l'ODEM, de Quensière et al., etc., en sont plusieurs repères parmi d'autres. Leurs observations et analyses ont été abondamment utilisées pour construire le modèle: en cela, l'entreprise a rempli son objectif, qui était aussi de proposer une synthèse de plusieurs résultats scientifiques.

\section{Paysages et unités de production économiques: les mosaïques invisibles}

Toutes les entités spatiales du modèle sont la résultante de plusieurs petits espaces définis par la microtopographie et la végétation, et qui constituent la mosaïque géographique des écosystèmes inondables (Amoros et Petts, 1993). Une telle unité paysagique, de l'ordre de la dizaine à la centaine d'hectares en dimensions, est produite par les modelés hydriques sur plusieurs millénaires et l'exploitation par l'homme. Un chenal contient et intègre: des bourrelets de berges, des chenaux de plus petite dimension, les tressages alluviaux et les bras morts (ou temporairement en eau) des tracés antérieurs, des mares permanentes et des mares temporaires; des villages (qui contiennent eux-mêmes plusieurs paysages et espaces fonctionnels), des habitats temporaires, des pêcheries de différente nature selon les engins et les calendriers autorisés, des pâturages différemment gérés selon le statut ${ }^{10}$ de leurs propriétaires, des sols non productifs (piétinement, encroûtements de surface, surpâturage) ; des espaces boisés, des vétivéraies, des bourgoutières, des oryzaies; des rizières de bas-fonds profonds, des rizières plus élevées; des sections profondes et des hauts-fonds dans les chenaux, des bancs de sable émergents en étiage; des lieux sacrés, des cimetières, des espaces réservés ou protégés... Les plaines et les lacs représentent des mosaïques plus simples.

Les multiples éléments de ces mosaïques peuvent être interprétés en termes de paysage, d'unités phyto- ou pédo-écologiques, d'utilisation du sol; ils sont identifiables sur le terrain et sur les photographies aériennes, ce qui a permis de donner à chacune des zones géométriques du modèle des attributs et donc un contenu implicite mais détaillé et précis.

Les groupes d'acteurs qui dirigent le processus de production dans le modèle réunissent plusieurs unités familiales de même catégorie socioprofessionnelle recensées dans la même entité spatiale. Il n'existe pas dans le delta rural de communauté de production qui soit stable en nombre et homogène en contenu, dans l'espace et dans le temps, en même temps que statistiquement identifiable. Les aînés sont les ayants droit aux moyens de production et décident d'une partie du processus de la production. Les jeunes hommes sont les principaux actifs de la production de bétail et de poisson; chez les agriculteurs, ce sont toutes les classes d'âges et les deux sexes qui participent aux travaux. Les communautés de production s'emboîtent en marmites (ceux qui mangent ensemble), en concessions, en quartiers, en villages ou campements, qui contiennent des effectifs et des proportions très variables d'actifs et de non actifs. L'"unité de production" utilisée en économie n'est pas clairement identifiable ici puisqu'elle se segmente et se regroupe selon des critères liés en partie - mais en partie seulement - à la

10. Clan familial de tradition peule ou bien communauté villageoise issue d'une colonisation agricole bambara, par exemple. production. Cette mobilité et la variété des stratégies individuelles et familiales n'ont pas permis de construire un modèle de stratégie applicable de façon cohérente. Pour cette raison, ce sont des modèles de comportement spatial (déplacements de maind'œuvre, transhumance pastorale) qui ont été écrits. 
Pour la pêche et la riziculture, la solution retenue a été de représenter des groupes de 20 producteurs actifs réels ${ }^{11}$, projetés sur les zones géométriques du delta à partir des données par villages du Recensement général de la population et de l'habitat du Mali (1987, mis à jour 1998).

Le problème est compliqué pour l'élevage, par la dissociation géographique entre les propriétaires des animaux (sédentaires des gros villages du delta et souvent extérieurs au delta) et les bergers (mobiles, recensés dans les villages du delta et hors du delta, fréquemment différents des précédents). On a vu plus haut que, dans le modèle, les acteurs de la production pastorale ne sont ni les premiers ni les seconds. La solution retenue est fondée sur la notion de troupeau (Gallais, 1967; CIPEA-ODEM, 1983), dont le contenu (variable en nombre et en composition) est lié à l'histoire des clans éleveurs. Chaque troupeau est décomposé ici en groupes de 100 têtes, rapportés aux zones correspondant à leur secteur d'entrée dans le delta, en octobre-novembre.

\section{Des échelles et des niveaux d'organisation spécifiques}

Au-delà des possibilités de simulation et de leur pouvoir intégrateur, le principal résultat de cette opération de modélisation parait résider dans un système cohérent d'échelles et de niveaux d'organisation correspondant aux processus hydrologiques, biologiques et socio-économiques. C'est ce système qui peut maintenant servir de base à une politique d'aménagement, ou à de nouvelle études interdisciplinaires. Ce système est caractérisé par:

- un niveau de granularité moyen; échelle et organisation spatiale: 100 unités spatiales pour représenter un écosystème de $30000 \mathrm{~km}^{2}$; échelle temporelle: 3 ans, 15 jours; - un mode de représentation du comportement des acteurs socio-économiques: le modèle représente le comportement de groupes d'agents et non pas leur stratégie; ce choix est lié au niveau de granularité;

- un mode spécifique de représentation des processus spatialisés dans lequel l'évolution physique des unités spatiales conditionne les comportements des agents qui y évoluent, alors que l'on choisit plus généralement de définir le comportement spatial des agents à partir de la représentation qu'ils ont de l'espace dans lequel ils évoluent.

Il reste à se poser la question de la signification de ces choix et de leur généralisation à l'étude de l'évolution des écosystèmes exploités et de leur gestion.

\section{Références}

Amoros C., Petts G.E. dir. (1993). Hydrosystèmes fluviaux. Paris: Masson, 300 p.

BARRIÈre O., BARRIÈre C. (1995). Le Foncier-environnement: pour une gestion viable des ressources naturelles renouvelables au Sahel. Répertoire des conflits fonciers du delta intérieur du Niger (Mali). Montpellier: Orstom, tome 2, n.p.

Benga E. (2000). Modélisation intégrée du Delta intérieur du Niger: le manuel de l'utilisateur.

CIPEA-ODEm (études réalisées par) (1983). Recherche d'une solution aux problèmes de l'élevage dans le delta intérieur du Niger au Mali. Bamako-Addis-Abeba: Ministère chargé du Développement rural, 5 vol., 1100 p., cartes h.t.

FAY C. (1989). "Sacrifices, prix du sang, "eau du maître”: fondation des territoires de pêche dans le Delta Central du Niger (Mali)» et "Systèmes halieutiques et espaces de pouvoir: transformation des droits et des pratiques de pêche dans le Delta Central du Niger (Mali), 1920-1980 ». Cahiers des Sciences Humaines, vol. 25, nº 1-2, p. 159-176 et 213-239.
11. Les producteurs actifs réels représentent environ un cinquième de l'effectif total de la population rurale dans le delta. 
FAY C. (1997). "Groupes et territoires au Maasina: logiques de contrat et logiques de la force », in J. Bonnemaison, L. Cambrézy, L. Quinty-Bourgeols, dir. Le Territoire, lien ou frontière. Paris: IRD. CDROM, fichier FAY.PDF, 9 p.

Gallais J. (1967). Le Delta Intérieur du Niger, études de géographie régionale. Dakar-Paris: Larose, mémoire IFAN nº 78, 2 vol., $621 \mathrm{p}$.

Gallais J. (1984). Hommes du Sahel, espaces-temps et pouvoirs, le delta intérieur du Niger, 1960-1980. Paris: Flammarion, $289 \mathrm{p}$.

Kelly S., Kuper M., Marieu B. (1998). Rapport de mission Delta Intérieur du Niger, 10-23 novembre 1998. Bamako: IER/IRD, $19 \mathrm{p}$.

KUPER M. (1997). Irrigation management strategies for improved salinity and sodicity control. Thèse de doctorat, Université Agronomique de Wageningen, Pays-Bas.

Kuper M., Mullon C., Poncet Y., Orange D., Morand P. (1999). Modélisation intégrée d'un écosystème inondé et gestion des eaux: le cas du delta intérieur du Niger au Mali. Actes des Journées scientifiques FRIEND-AOC-ZTH-AAH, (séminaire sur les « Ressources en Eau de l'Afrique Occidentale et Centrale »). Yaoundé, Cameroun, 30 novembre-2 décembre 1999. Paris: Unesco.

Kuper M., Mullon C., Poncet Y., Morand P., Orange D., Arfi R., Mahé G., Benga E. (2000). «Modélisation intégrée d'un écosystème inondable: le delta intérieur du Niger au Mali ». Séminaire international Gestion intégrée des ressources naturelles en zones inondables tropicales, 20-23 juin, Bamako, Mali, 19 p., à paraître IRD.

LAË R. (1992). «Influence de l'hydrologie sur l'évolution des pêcheries du delta central du Niger de 1966 à 1989 ». Aquatic Living Ressource, $\mathrm{n}^{\circ}$ 5, p. 115-126.

Maiga O., Kuper M., Gosse-Healy B. (2000). "La riziculture traditionnelle autour de la mare de Débaré dans le delta intérieur du Niger au Mali ». Séminaire international Gestion intégrée des ressources naturelles en zones inondables tropicales, 20-23 juin, Bamako, Mali, 14 p., à paraître IRD.

MARIE J. (2000). «Milieux, riziculture, infrastructure pastorale: les enjeux spatiaux et fonciers dans le delta intérieur du Niger (Mali)». Séminaire international Gestion intégrée des ressources naturelles en zones inondables tropicales, 20-23 juin, Bamako, Mali, 20 p., à paraître IRD.

MARIE J. (2001). DELMASIG: Hommes, milieux, enjeux spatiaux et fonciers dans le delta intérieur du Niger (Mali), HDR Université Paris-X, 3 vol., 291 p., cartes h.t.

MARSILY G. de (1997). "De la validation des modèles en sciences de l'environnement », in F. Blasco dir. Tendances nouvelles en modélisation pour l'environnement, p. 375-382.

Poncet Y. (2000). «Une lecture temporelle de la pêche au Mali », in Poncet Y., dir. Les Temps du Sahel. Paris: IRD, p. 81-108.

PONCET Y. (2000). «Le système halieutique du delta central (Mali) : structurations de l'espace et cohérence des articulations fonctionnelles ", in Gascuel G., Chavance P., Bez N., Biseau A., dir. Les Espaces de l'halieutique. Paris: IRD, p. 549-567.

Poncet Y., M. Kuper, C. Mullon, P. Morand, D. Orange (2001). «Représenter l'espace pour structurer le temps: La modélisation intégrée du delta intérieur du Niger au Mali ", chap. 7, in Représentations spatiales et développement territorial, Lardon S., Maurel P., Piveteau V., dir. Paris : Hermès, p. 143-162.

Poncet Y., Orange D. (1999). «L'eau, moteur de ressources partagées: l'exemple du delta intérieur du Niger au Mali ». Aménagement et Nature, n²132, p. 97-108.

PONCET Y., Quensière J. (1996). «Analyse des organisations spatio-temporelles, étape essentielle à la conception d'un SIG : l'exemple des pêcheries artisanales du delta central du Niger », in CHRISTOPHE C., LARdon S. et MoneStiez P., dir. Étude des phénomènes spatiaux en agriculture. Paris : INRA, p. 15-31.

QUENSIÈRE J., dir. (1994). La Pêche dans le delta central du Niger. Paris: IRD-Karthala-IER. 2 vol., 495 p. et cartes h.t.

Quensière J., Poncet Y., Fay C., Morand P., Kassibo B., Rey H., Baumann E., Bénech V., Bousquet F., Dansoko D., Herry C., Lä̈ R., NiarÉ T., Raffray J., Troubat J.J., Weigel J.Y. (1994). "Synthèse et recommandations », chapitres 6.1, 6.2, 6.3, 6.4. et 6.5, in QueNSIĖRE J., dir. La Pêche dans le delta central du Niger, p. 409-446. 